\title{
ㄷำ1
}

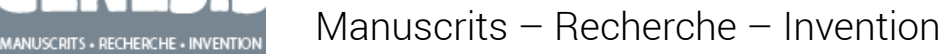

$50 \mid 2020$

Aragon

\section{Un fonds, son legs}

\section{Renate Lance-Otterbein}

\section{OpenEdition}

\section{Journals}

Édition électronique

URL : https://journals.openedition.org/genesis/5197

DOI : 10.4000/genesis.5197

ISSN : 2268-1590

\section{Éditeur :}

Presses universitaires de Paris Sorbonne (PUPS), Société internationale de génétique artistique littéraire et scientifique (SIGALES)

\section{Édition imprimée}

Date de publication : 15 juillet 2020

Pagination : $39-54$

ISBN : 979-10-231-0679-4

ISSN : $1167-5101$

\section{Référence électronique}

Renate Lance-Otterbein, « Un fonds, son legs », Genesis [En ligne], 50 | 2020, mis en ligne le 15 juillet 2021, consulté le 03 septembre 2021. URL : http://journals.openedition.org/genesis/5197 ; DOI : https://doi.org/10.4000/genesis.5197 


\title{
Un fonds, son legs
}

\author{
Renate Lance-Otterbein
}

L a naissance du fonds Aragon survient en l'absence de signes annonciateurs. Créateur d'avant-gardes, l'écrivain récuse l'enfermement de ses manuscrits dans un lieu de conservation. Communiste convaincu, il envisage de confier ses archives à son parti avant de décider de les remettre «à la nation française» et de désigner le CNRS comme propriétaire et son laboratoire de critique génétique, le Centre d'histoire et d'analyse des manuscrits modernes (CAM, prédécesseur de l'ITEM), comme dépositaire de son legs ${ }^{1}$.

Lors de l'inauguration au CNRS, Aragon rappelle le déroulement :

C'est avec une grande discrétion que l'un des vôtres, M. le Directeur général, m'avait, [...] je dirais : sondé, pour savoir si j'étais prêt à fournir aux chercheurs [...] les éléments jusqu'à présent fort occasionnellement utilisés pour la compréhension de ce que je puis avoir écrit au cours de ces dernières soixante années $[\ldots]^{2}$.

Le projet coïncide, certes, avec le souci de l'octogénaire et veuf ${ }^{3}$, mais il met «près d'un an de réflexions et d'approches ${ }^{4} »$ avant d'aboutir. Aragon n'est pas l'instigateur 5 et son «désir» de faire ce don doit mûrir. La constellation culturelle, historique et politique, quelques années après la signature du Programme commun de gouvernement de la gauche française, est favorable : la notoriété de l'écrivain est telle et son œuvre à ce point importante que des personnes compétentes et influentes interviennent en faveur de sa sauvegarde. À l'instar du

1. «Aragon remet à la nation française, quelle que soit la forme de son gouvernement, le legs littéraire d'Elsa Triolet et l'ensemble des manuscrits et documents en sa possession qui appartiendront au Centre national de la recherche scientifique [...]», contrat du legs, «fait à Paris, le 22 juin 1976 », signé «L. Aragon».

2. Louis Aragon, «Un grand art nouveau : la recherche», manuscrit Cardoze [désormais ms], $\mathrm{f}^{\circ} 4$

3. Héritier d'Elsa Triolet, décédée en juin 1970, et sans enfants, Aragon confiera ses affaires à Jean Ristat.

4. $\mathrm{Ms} \mathrm{f}^{\circ} 1$. Ce délai pourrait correspondre à la signature du contrat. Pour l'essentiel, les affirmations sont confirmées par les protagonistes et d'après les communications de M. Apel-Muller, à l'époque maître de conférences à l'Université de Franche-Comté et spécialiste d'Elsa Triolet, et de Jean Ristat, en sa qualité de collaborateur et héritier d'Aragon, interrogés respectivement en 1986, 1992 et 1994. Le contact avec Louis Hay, directeur de recherche, fondateur et directeur du CAM, à l'époque membre du Comité national du CNRS, aurait été établi par Jean Peytard, professeur à l'Université de Franche-Comté (peut-être à la demande de son collègue M. Apel-Muller), lors d'une soutenance de thèse, le 3 mars 1976 (communication écrite du 28 juin 1995). Louis Hay dit conserver des notes antérieures à mars 1976 et estime la durée des pourparlers à trois ou quatre années (communication orale du 26 avril 1994). Dans un premier temps, il devait s'agir d'établir les microfilms des manuscrits. On envisagea ensuite de constituer un fonds de manuscrits pour assurer l'avenir de l'héritier (communications de Louis Hay et de Jean Ristat).

5. Ses manuscrits de jeunesse se trouvaient au fonds Doucet. En 1965 il avait prévu de remettre au premier établissement national de conservation des documents qui concernaient les rééditions des Voyageurs de l'impériale (voir Euvres romanesques croisées d'Elsa Triolet et Aragon [désormais ORC], Robert Laffont, t. XV, 1972, p. 28) et avait offert à la Bibliothèque nationale un ensemble manuscrit détaché du Roman inachevé, à l'occasion de l'exposition sur Elsa Triolet en 1972. 
donateur qui bouscule, révolutionne et déborde le cadre convenu de la création, le fonds Elsa Triolet-Aragon, encore en gestation, transgresse déjà les conventions institutionnelles ${ }^{6}$.

Quelles sont alors les volontés du légateur?

Son allocution lors de la cérémonie inaugurale 7 du 4 mai 1977 au CNRS en trace les contours ${ }^{8}$. On examinera plus loin quelques points forts, à la lumière de déclarations antérieures. En dépit de digressions, suspensions et détours fréquents, le souffle profond de ce discours a l'accent des occasions solennelles. Son propos n'en est pas moins concret, rassemble les arguments favorables à la réalisation du projet qui avaient convaincu l'orateur, leur adjoint ses propres réflexions et dresse des prescriptions et proscriptions. Aragon, tout en soulevant des questions requises par les circonstances (entre autres, qu'est-ce que la recherche et le CNRS, que faut-il entendre par «manuscrit» et quel usage peut-on en faire, quelles sont ses approches, quelles sont les prérogatives de l'auteur, quelle est la place de la littérature et de la critique ?), présente son futur fonds comme un objet à créer, avec des documents à réunir par lui-même, et par son héritier. Il rend compte des divers emplois du manuscrit, oppose la critique à la création dont il proclame la suprématie et se revendique de la recherche. Il réclame sérieux et discrétion à l'égard des archives et de sa personne. En communiquant ses «manuscrits à l'étude de ceux qui [peuvent] se poser des questions», Aragon dit inaugurer une «démarche sans précédent» et ouvrir de nouvelles perspectives à la recherche. Ce faisant, il veut «tendre la main» à d'autres auteurs et souhaite que son geste de donateur fasse école. Quant à la motivation du legs - «rien ne pouvait être plus voisin de mes désirs 9 » - elle se résume ainsi : faire comprendre l'histoire et la culture du Xxe siècle.

Et voici qu'aujourd'hui à la demande du C.N.R.S. je me trouve devant vous, au printemps de 1977, ayant accepté le travail à proprement parler ÉNORME, c'est-à-dire hors de toute norme, de ramasser, rassembler, les écrits de toute ma vie, c'est-à-dire les manuscrits de toute ma vie d'écrivain, avec leurs étapes diverses, toutes les versions que j'en puis retrouver, du manuscrit proprement dit à sa transformation en livre... ou même plus, puisqu'il m'arrivera d'y ajouter ce qui n'est entré dans aucun de mes livres... Je m'en suis expliqué [...] pour montrer que mon souci ne se bornait pas à mes propres écrits... Suis-je fou? Peut-être... Mais, pour le plaisir d'un cadeau, allais-je donc me priver d'être ce que je fus tout le long de ma vie consciente? C'est-à-dire un écrivain, qui toujours poursuit sa tâche d'écrivain, sans tenir compte des années, et pourtant ne fallait-il pas envisager l'aléatoire de l'âge 10 ?

6. Au CNRS, qui n'a pas vocation à conserver des archives, le fonds ET-A sera une exception statutaire et administrative, ce qui compliquera son existence au sein de cette institution. Mais c'est un autre chapitre de son histoire.

7. Aragon remet une boîte d'archives symbolique lors de cette cérémonie (au siège du CNRS à Paris, 15 quai Anatole-France) initialement prévue le mercredi 9 février 1977 à 18 heures, mais dont la date fut déplacée sous un prétexte fallacieux. À ce propos, Aragon ne cache pas son agacement : «C'était un moment, où - paraît-il - le CNRS avait été dans l'obligation de retarder la tenue de la cérémonie qui prend place ici et aujourd'hui. Sans entrer dans le détail de l'affaire, je n'ignorais pas qu'il y avait des gens qui s'imaginaient alors que j'allais prononcer un discours politique, lequel viendrait bien malencontreusement s'insérer dans la campagne des élections municipales [...] Mais nous voilà réunis ici, et vous pouviez [...] juger [...] de la légèreté assez enfantine de certaines têtes politiques» $\left(\mathrm{ms} \mathrm{f}^{\circ} 15\right)$.

8. Cette réflexion, menée il y a plus de vingt ans, a été remaniée et calibrée pour cette publication.

9. $\mathrm{Ms} \mathrm{f}^{\circ} 4$.

10. $\mathrm{Ms} \mathrm{f}^{\circ} 30$. Voir aussi la version manuscrite partielle, fig. 1. 


\section{$\mathrm{Cor}$}

le nom alle t apraraithe dans ce premir volu e de ma forlie

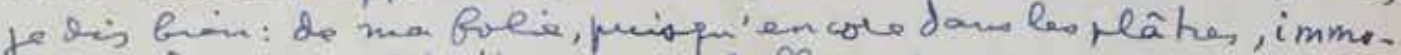
filige' Sans mon lit, d'avas, our': folloment, accepté d'écris

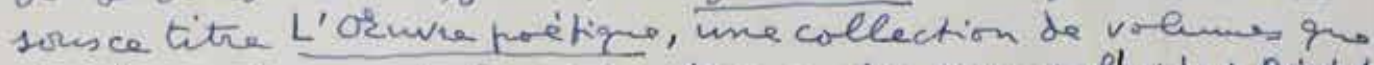

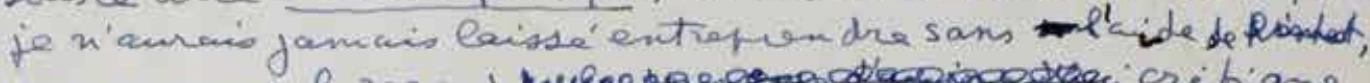

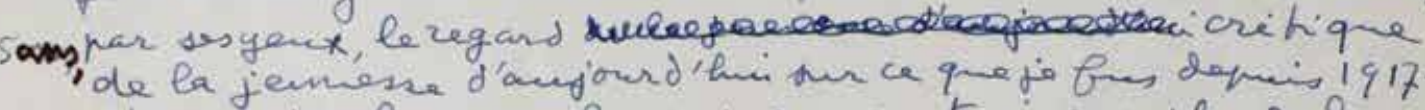
jecturese d'anjourd thi tur ce qwe jo fus dopis 1917. et sans, dans be fixvolumes parus en trpis ans, flus de dax

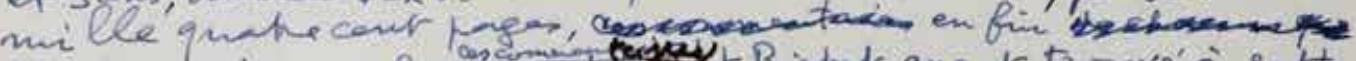

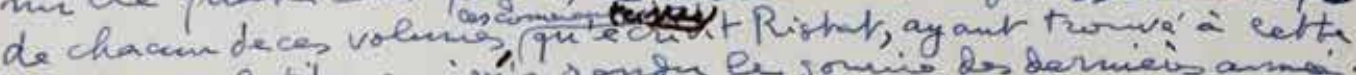

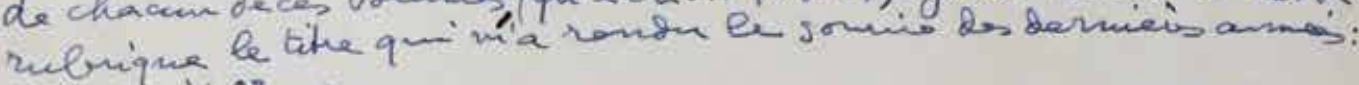
Hors d'oruve...

Ef voici qu'anjourd hui, sur la demande de C.N.R.S., je mépturuvé devant vours, an prinlemp de 1977 , ayant accepté latravail à poprement porlen ENORME, c'astiaidio hous de tante norme, de ramasrer, rassombler, besécits de

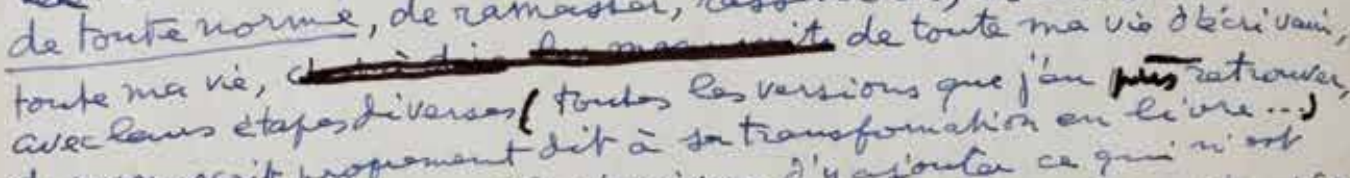

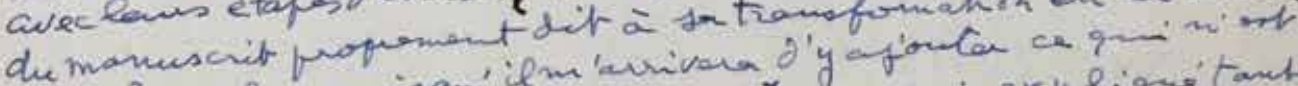
on môme ples, prisq a' hes lives... Xe mian suis explique tantiot,

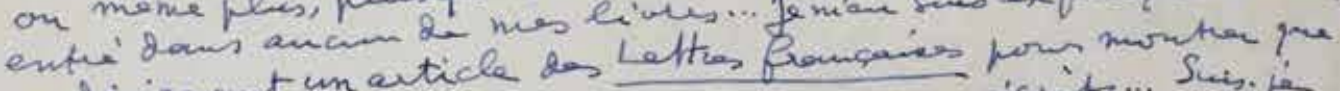

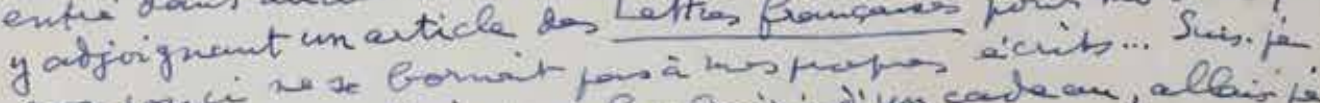

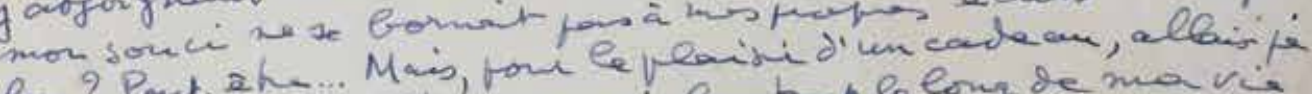
fou? Pout. ah... M' ithe ceque jofus toun le long de ma vi:

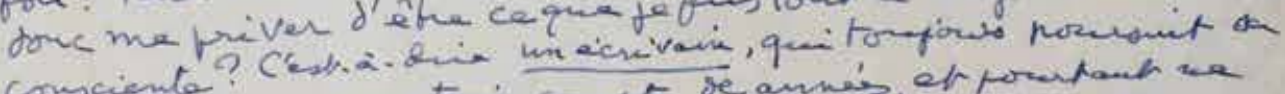

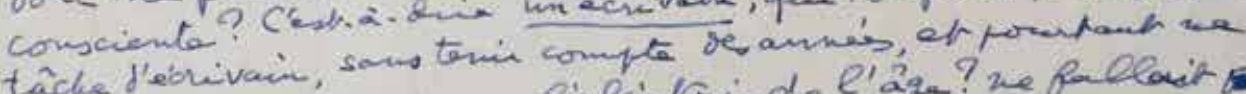

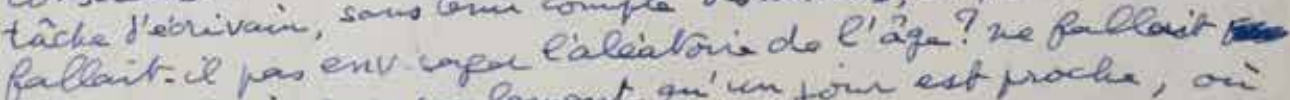
- il pes peevori, non soulament qu' un foir est proche, ori

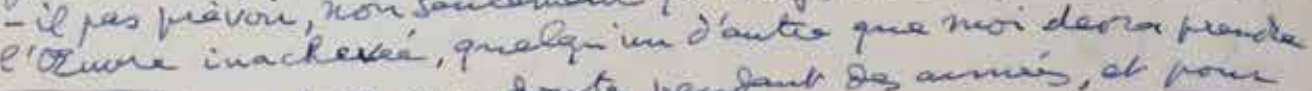

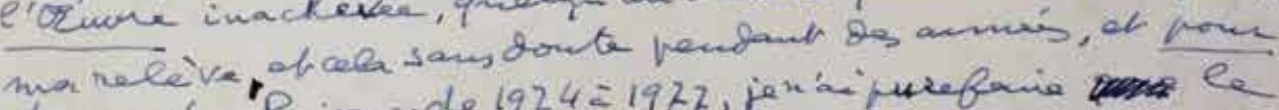

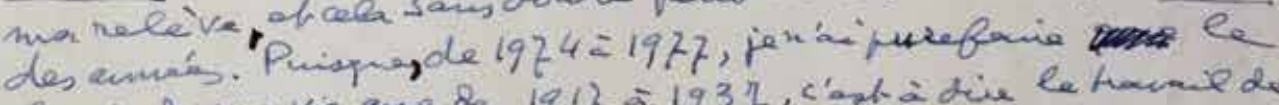
chemin de ma vis que de 1917 a $\lg 37$, c'asta a dive le tharwe da

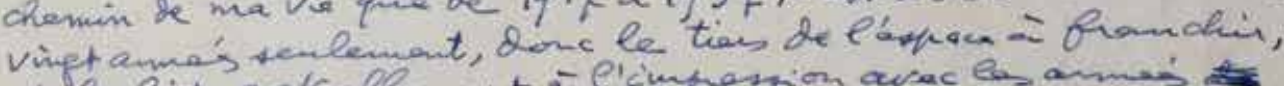

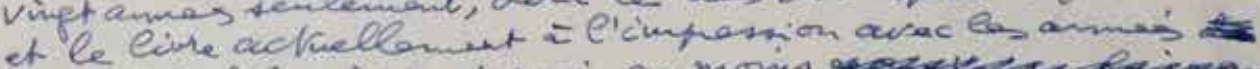

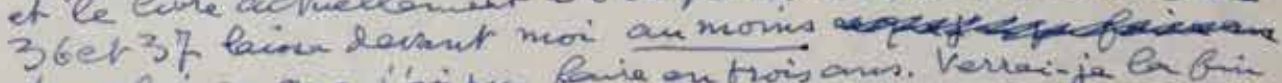

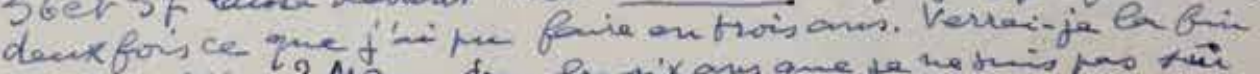

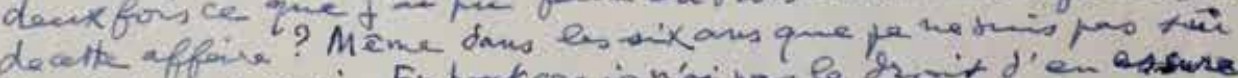

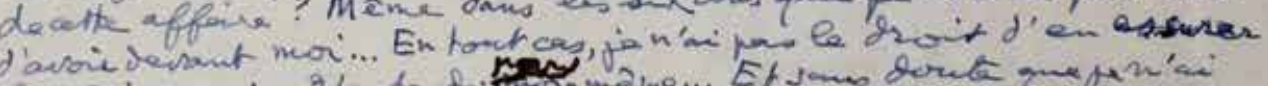

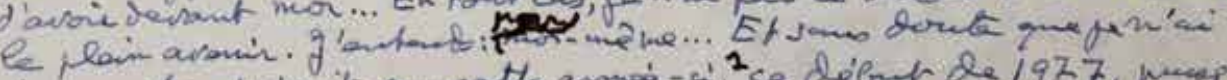

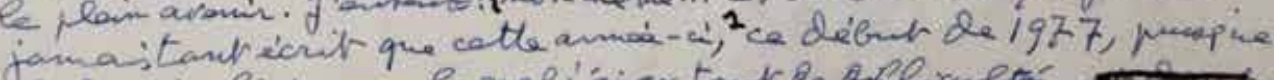

\section{C'est deritalins.}

Fig. 1 : Allocution au CNRS, 4 mai 1977, version manuscrite partielle avec reprises tardives, $\mathrm{f}^{\circ}$ 31/5. BnF, Mss, fonds Aragon. () Paris, BnF. Avec l'aimable autorisation de Jean Ristat 


\section{Transmission du texte}

\section{Art versus recherche}

Il existe plusieurs versions de l'allocution au CNRS :

- Publiée le lendemain dans L'Humanité11, elle sera reprise dans les Essais de critique génétique «d'après le discours prononcé 12 » avec des changements mineurs et des suppressions ${ }^{13}$. - Une version manuscrite partielle et mêlée à d'autres fragments tardifs qui, eux, s'orientent vers d'autres horizons, est conservée au fonds Aragon (fig. 1 à 3). Des variantes induisent une reprise ultérieure à l'événement pour ces feuillets comprenant une page de titre et sous-titre définitifs.

- De son vivant, le poète Pierre Lartigue détenait la photocopie d'un manuscrit complet (trente-deux folios, sans le titre définitif), sauvé par Michel Cardoze, journaliste chargé de la publication par L'Humanité. Le graphisme ressemble à l'écriture tardive d'Aragon. Ce dernier s'est-il servi du gros stylo-feutre noir, apte à tracer des grandes lettres pour yeux sans lunettes ${ }^{14}$ ? Malgré la grosseur des lettres, un feuillet A4 compte jusqu'à une bonne quarantaine de lignes. La marge gauche est réservée, non la droite, les italiques sont marqués par soulignement et «ital.» dans la marge. Les corrections essentiellement supra-linéaires 15 améliorent ou rectifient l'expression ou la syntaxe. Ce «manuscrit Cardoze» est ici cité en référence.

Le titre de l'allocution «Un grand art nouveau : la recherche» vise d'emblée une mise en valeur de la recherche par l'art. Cette recherche que le sous-titre, «Il faut savoir aider les chercheurs», flatte avec empathie, mais que la citation de l'intertitre, «Une gifle majeure à tous ceux qui ne croient pas à l'écriture ${ }^{16}$ », transforme en un hymne à l'art (littéraire) aux dépens de la science.

Puis, dès l'introduction apparaît une «suspicion» à l'égard de recherches «indiscrètes » et «diversement regardées [...] notamment par les écrivains» qui se verraient confrontés à «des inquisitions»17. Par la suite, il y aura quelques mises au point dont la rectification d'un communiqué de presse erroné et l'allusion aux mérites du donateur, son sens de la

11. L'Humanité, 5 mai 1977, p. 1, 8, 9. Le journal annonce le «texte intégral» et suit le manuscrit à la lettre, mais ne reproduit que quelques lignes de la partie «Le quatrième chant», reprise d'un article paru dans Les Lettres françaises $\left(n^{\circ} 1370,27\right.$ janvier 1971) et consacré aux jeunes poètes Lionel Ray, Marc Delouze, Michel Cahour ainsi qu'au peintre Alain Le Yaouanc.

12. Essais de critique génétique, édités par le CAM, Flammarion, 1979, p. 5-19. (Je souligne.)

13. Changements de syntaxe et ponctuation. Deux suppressions importantes : les parties intitulées «Le quatrième chant», reprise d'un article des Lettres françaises ( $n^{\circ} 1370,27$ janvier 1971), et «Post-scriptum », consacré à Jean Ristat (voir infra, «La phrase sans fin»). Deux modifications : de la première phrase, «Vous êtes avant toute chose, M. le Directeur général, à la tête d'un organisme qui donne enfin légitimité à toute une catégorie de travailleurs intellectuels d'un type nouveau, lesquels se sont appelés eux-mêmes les chercheurs, et sous l'aile des universités, ont entrepris depuis un certain nombre d'années de donner corps à leurs recherches [...]» (ms $\left.\mathrm{f}^{\circ} 1\right)$, les éditeurs du CAM ont remplacé «Vous êtes [...] M. le Directeur» par «Le CNRS est un organisme». Un autre paragraphe, qui apostrophe «M. le Directeur général» et l'assimile à la presse peu sérieuse, est supprimé.

14. De ce graphisme, on trouvera un échantillon dans le manuscrit reproduit en fin d'article. Ici les caractères, de plus petit format, sont limités par la taille du support papier (feuillet de $20 \times 14,8 \mathrm{~cm}$ ).

15. Environ 70 sur 32 folios, soit une moyenne de deux par feuillet, autant de corrections sur le recopiage du «quatrième chant» que sur le texte nouvellement rédigé. L'ensemble serait donc une mise au net.

16. L'Humanité, 5 mai 1977 , p. 9 et $\mathrm{ms} \mathrm{f}^{\circ} 32$.

17. Ms $\mathrm{f}^{\circ} 1$. Sur le manuscrit le mot chercheur est presque toujours souligné pour être imprimé en italiques. 
responsabilité politique, donc des remarques témoignant d'une certaine irritation et qui se résument ainsi : le donateur ne se laisse pas impressionner par la recherche, et l'honneur revient à la littérature.

Aragon renoue ici avec un de ses sujets anciens. En 1959, il avait relevé l'évolution sémantique du mot recherche : «personne ne l'applique plus [...], qu' aux gens de la recherche scientifique », alors «qu'au siècle dernier [...] on ne l'appliquait qu'aux chercheurs d'or ». Il est vrai qu'Aragon avait déjà une trentaine d'années quand l'acception actuelle du mot est entrée dans l'usage courant. Et encore plus tard, il a vu naître le CNRS, alors que lui s'intéresse depuis longtemps «assez passionnément à cette autre catégorie nouvelle de "chercheurs", dont la recherche est un or intellectuel singulier [...] leur travail personnel vaut par cela qu'il s'insère dans le travail de tous les autres, et qu'à eux tous ensemble, les romanciers d'aujourd'hui et de demain [...] travaillent comme des artisans dans la cathédrale à des détails descriptifs, dont la juxtaposition donnera à ce siècle une somme, donnera à ce siècle une survie inconnue avant lui 18 .»

Dans cette «cathédrale» réside la conception de l'art et de la littérature d'Aragon, une variante consensuelle du «réalisme socialiste». La littérature, manifestation d'invention donc d'originalité, incarne la nouveauté : elle assimile les connaissances, voire même les anticipe. L'œuvre survit à son auteur mortel et existera toujours car le mythe est éternel. Pacificatrice et humanisante, la création littéraire rivalise avec les plus hautes instances de l'esprit 19 : «Notre tâche [de romancier] est [...] le grand art des temps nouveaux, et il nous faut savoir viser aussi haut et plus loin si possible que la science même 20 .»

Résultat de plusieurs décennies de combats idéologiques gagnés par Aragon, son appel à rivaliser avec la science avait produit des formulations semblables dans les officielles prises de position culturelles du PCF (dont la résolution du Comité central d'Argenteuil, 196621). De ce fait, l'abandon du dogme réaliste entérine une ouverture culturelle qu'Aragon appelait de ses vœux, voire imposait à son parti22. Onze ans plus tard, devant le CNRS, l'argumentaire du légateur témoigne donc toujours de cette même ambition pour la littérature : être supérieure aux disciplines voisines, sciences comprises.

Vis-à-vis des chercheurs réunis, qui auront à étudier son fonds, le légateur réclame son droit à la «critique de la critique ». En effet, le contrat du legs prévoit que le premier traitement des manuscrits se fera «immédiatement sous [s]a direction »-Aragon saura surveiller les résultats, c'est ce qu'à l'époque, il envisageait sans doute. En d'autres occurrences, Aragon se méfie des théoriciens de la littérature, se comptant, lui créateur, parmi ceux qui «fondent leur pensée et leur action sur l'unité, la non-séparation de la théorie et de la pratique ${ }^{23}$.»

18. Louis Aragon, J'abats mon jeu [1959], désormais J'abats..., Paris, Mercure de France, 1992, p. $21,22$.

19. Argument avancé depuis «Un roman commence sous vos yeux », 1937.

20. J'abats..., op. cit., p. 276.

21. Je renvoie à mon article «La longue marche vers Argenteuil ou le droit du créateur à la recherche dans les textes d'Aragon» dans Aragon et le Comité central d'Argenteuil, Annales de la société des amis de Louis Aragon et Elsa Triolet, $\mathrm{n}^{\circ} 2,2000$, p. 155-167.

22. En 1966, cette ouverture s'inscrit dans la perspective de l'Union de la gauche.

23. «Je me méfie assez des théoriciens de la littérature et c'est pourquoi j'hésite à prendre place à côté d'eux... », «Discours de Prague», Les Lettres françaises, n 944, 20 septembre 1962, et Recherches internationales, juilletaoût 1963 , p. 45. 
Dans cette unité s'enracine pour lui le fondement de la communauté nationale, c'est-à-dire «le concert où élites et masses sont étroitement confondues». Et «de leur coexistence, de leur harmonie» serait faite la «grande culture humaine » 24 . Cette même mission culturelle est confiée au legs dont le contrat désigne de façon expresse la nation française comme destinataire. Dans cette conception aragonienne, la littérature est à la fois miroir, mémoire et chantier ouvert sur la culture d'un pays. Elle est son visage et son moyen de reproduction, de plaisir, son éros 25 .

De son côté, Jean Ristat verra dans l'acte du legs chez Aragon «le sentiment d'être utile, de servir "ce rêve modeste et fou". Il pouvait s'enorgueillir d'être lui aussi un chercheur et sa [...] donation ne procédait que de son désir de participer lui-même au travail en cours, en livrant les informations et éclaircissements de l'auteur, ce qu'il appelle "secrets de fabrication".

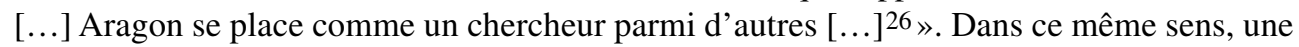
remarque de l'allocution adresse aux chercheurs un aparté de réconciliation :

Ce sont, tout autant que des ratures [...], ces modifications [...] qui risquent de produire $[\ldots]$ une méditation du lecteur, plus ou moins chercheur qu'il puisse être, devant le ou les textes proposés, et qui risquent le mieux de mettre, chercheur ou lecteur, le rêveur, de quelque sorte qu'il soit, sur la piste des secrets d'une création 27.

Ce seul argument accorde au chercheur le droit au «rêve», synonyme de création et invention, donc lieu de rencontre entre écrivain et critique, mais à condition d'observer cette prescription : faire preuve de sérieux et de discrétion.

\section{Sérieux et discrétion}

«Chez les uns», créateurs, «comme chez les autres, critiques, «la noblesse tient au sérieux du métier 28 », admet Aragon, et définit le niveau de son exigence : «C'est que toute la vie j'ai eu le goût de la perfection ${ }^{29}$. » Déjà dans J'abats mon jeu (1959), les «aveux » sur les «secrets de fabrication» étaient assortis de mises en garde : «ces armes que je vous donne cependant, craignez contre moi de vous en servir. 30 » Ailleurs, Aragon écrit : «Ceux qui se font un domaine de notre vie vont pouvoir à leur aise mentir. C'est cela, la mort : soudain la place faite au mensonge, le domaine du mensonge irrémédiablement agrandi ${ }^{31}$.»

Exigeant de la discrétion, Aragon vise le domaine privé, voire intime, mais donne comme exemple à ne pas suivre celui d'un chercheur qui «voulait savoir de [lui], en raison de [leur] amitié première, si oui ou non Pierre Drieu La Rochelle était ou n'était pas un impuissant».

24. Louis Aragon, La Culture et les Hommes, Paris, Éditions Sociales, 1947, p. 27 et 29.

25. J'abats..., op. cit., p. 112. «Les mots font l'amour avec le monde», ORC, t. II, p. 14.

26. «Aider à ce que cela survive», discours prononcé à l'occasion de la remise de mon Relevé d'inventaire du fonds au directeur général du CNRS, le 11 avril 1986, publié dans L'Humanité, 15 avril 1986, p. 22. Dans ce discours, Jean Ristat date le legs de 1975, donc un an avant la signature du contrat. Voir aussi note 4.

27. Ms f ${ }^{\circ} 9$.

28. $\mathrm{Ms} \mathrm{f}^{\circ} 3$.

29. Ms f $^{\circ} 26$

30. J'abats..., op. cit., p. 8,9 .

31. Louis Aragon, Jean Paulhan, Elsa Triolet, Le Temps traversé. Correspondance 1920-1964, Bernard Leuilliot (dir.), Paris, Gallimard, coll. «Les Cahiers de la NRF», 1994, p. 209. 
Enfreignant la bienséance, ce passage, qui poursuit sur le même ton, s'expose à la réprobation des éditeurs et sera supprimé des Essais de critique génétique.

Le désir de discrétion peut s'expliquer par la crainte d'être dessaisi du secret (des origines) ${ }^{32}$ ou des secrets (de fabrication), au risque de voir son œuvre amoindrie par des questions concernant l'auteur. Et la variante manuscrite serait suspectée de transcrire un lapsus révélateur, trahirait le(s) secret(s)?

En tant que romancier, poète, journaliste, directeur de rédaction et éditeur, Aragon voit d'abord dans le manuscrit le modèle destiné au «prote», l'objet servant à la fabrication de l'ouvrage, autorisé par l'auteur, adressé au public, d'où il tire sa consécration sociale, et qui lui confère une responsabilité morale. C'est pourquoi la variante manuscrite ne saurait se substituer au texte définitif et autorisé, mais prouvera, au contraire, que l'auteur a répondu le premier aux questions que se pose le critique ${ }^{33}$. L'écrivain réclame le respect de SA vérité du texte. Cette exclusive tire sa justification dans l'esthétique de l'œuvre finie, exprimée depuis les années trente.

À maintes reprises, l'allocution d'Aragon tourne autour du point central - de quoi le manuscrit est-il fait? -, le scrute, recule, l'encercle et produit à l'infini - et pour notre bonheur - ses variations sur le même thème :

Rien n'est plus orienteur (ou faudrait-il écrire orientant) que ces hésitations de l'écrivain devant son propre écrit, rien ne peut l'entraîner davantage à la variation que ces légères modulations de sa propre musique, ces retours dans les marges de soi-même, à quoi se reconnaît l'écrivain véritable. $[\ldots]$ cette dernière phrase est de celles où je me retrouve chez moi comme nulle part, précisément parce que la porte en est restée ouverte, où claque tout d'un coup le volet. Peut-être que tout ceci fait $[\ldots]$ que je me retrouve chez moi dans ce château magique du dire et du taire 34 .

Puis, débordé par sa virtuosité, Aragon recourt à nouveau à l'étymologie : «Le manuscrit, au sens premier du mot, peut bien présenter, cela est certain, des ratures, des substitutions de termes, des corrections en un mot, dont le tout est bien de savoir ce qui est l'état premier, second... ou définitif de la phrase ${ }^{35}$.» Enfin, surmontant sa réticence à exposer au grand jour son écriture en gestation, il s'approche de l'acception génétique : «Le mot manuscrit signifie l'état intermédiaire ou dernier de l'objet usuellement ainsi désigné par ce mot, mais aussi l'ensemble [...] des étapes qui mènent à la version définitive de l'œuvre ${ }^{36}$.»

32. Enfant non reconnu, il grandit cependant dans sa famille maternelle dont les membres sont présentés sous de fausses désignations. Aragon est à ce point traumatisé par son histoire et le tabou inhérent qu'il ne peut en parler simplement, directement et le propos de son interlocuteur est toujours à côté de la réalité, n'atteint jamais le noir de la cible car l'intensité du noir aragonien dépasse l'entendement. L'accès à l'auteur, au sujet est «barré», pour recourir à une formule lacanienne. Mais le drame personnel nourrit aussi l'écriture et le roman familial est distillé à petites doses dans 1'œuvre.

33. «[...] communiquer mes manuscrits à l'étude de ceux qui pouvaient se poser des questions [...] auxquelles l'auteur, les auteurs avaient répondu d'avance par la rature, la surcharge, la correction [...]» (ms $\mathrm{f}^{\circ}$ 4. Je souligne.) Voir aussi note 40.

34. Ms f ${ }^{\circ} 9$.

35. $\mathrm{Ms} \mathrm{f}^{\circ} 7$.

36. $\mathrm{Ms} \mathrm{f}^{\circ}$ 6. Voir aussi la version manuscrite partielle des figures 2 et 3. 


\section{**. \\ 棌 3 .}

Metter àla dissposition der cherchems la taxte, c'ashà dive le mamuorit decequelor a e'crit, celasuppose, il faut bia

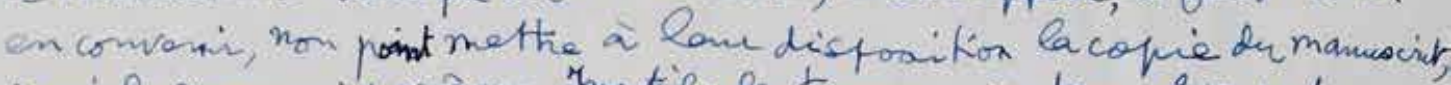
mais le mamacrit meme. Thutile de toumer an torn du por.

Il va sans dire que cela suppose ausar, le mannair liore', conie ettablic du dit manuserit pour a lecture. Cela w'st pas, serbats, l'apbaie on domnewr. Hest natrel, givil en demande la garoutie.

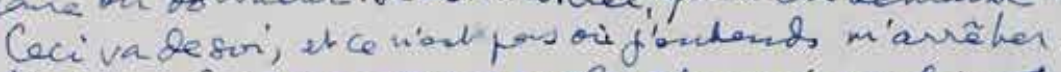

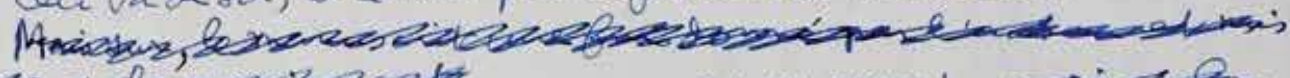

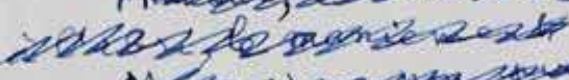

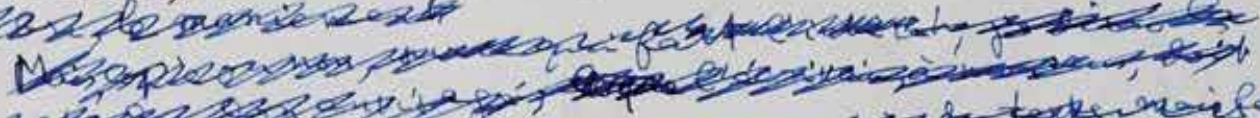

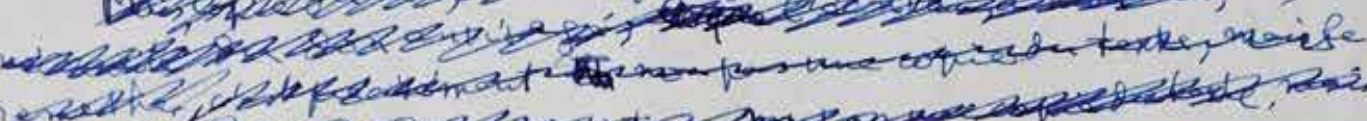

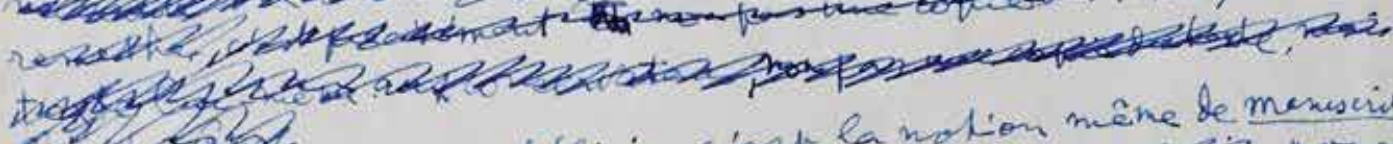
ce laxwast.

Ce ail fant ici définis, c'est la notion méme de manscirt

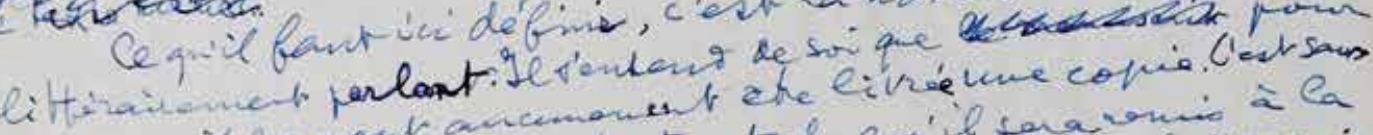

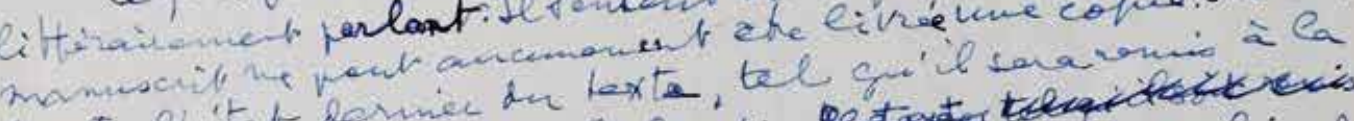

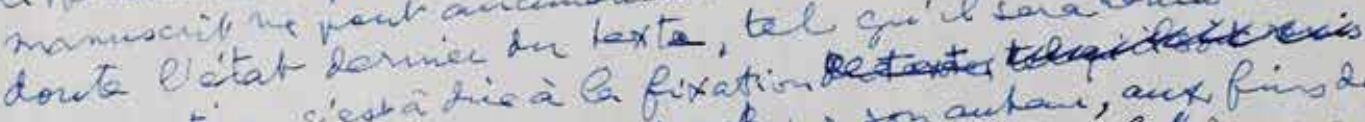
compursition, c'esta hic dudi fext per son autar, aux fins be

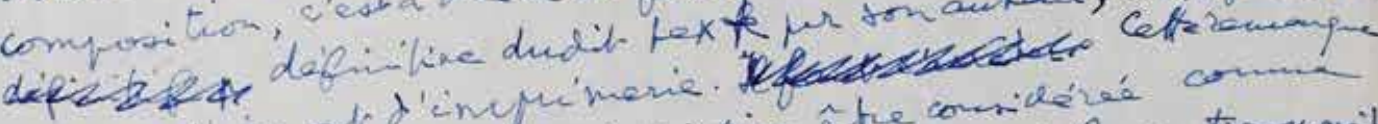

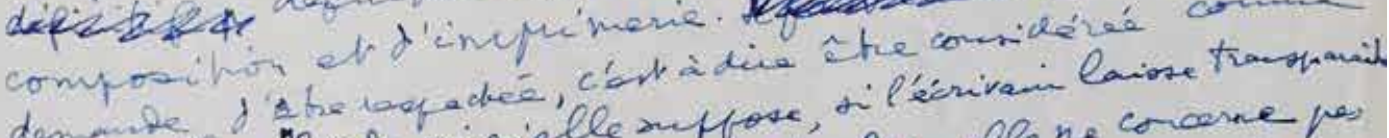

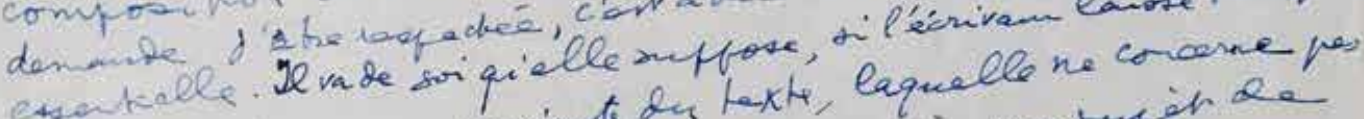

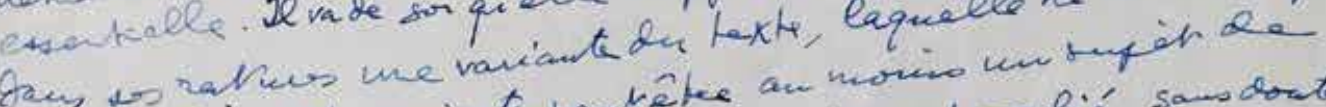

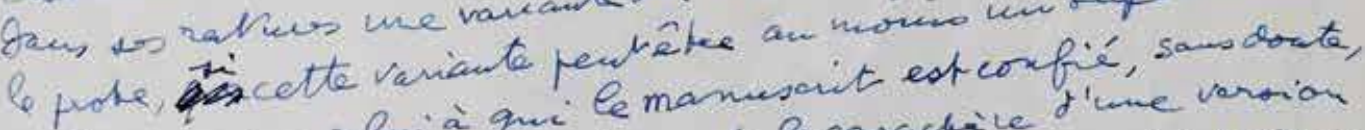
vifeexion pour celvi à qui le ma nusert est con d'une version

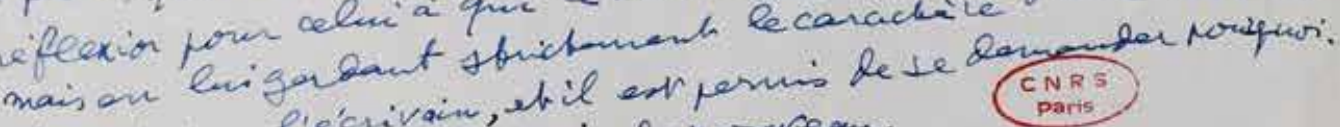
écarté per l'sécrivain, ehil en de korusear.

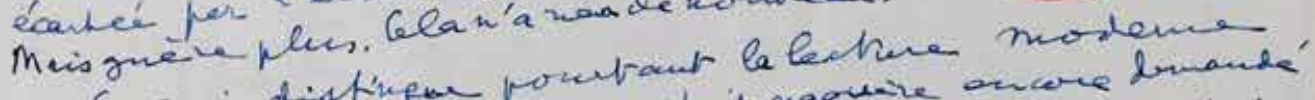

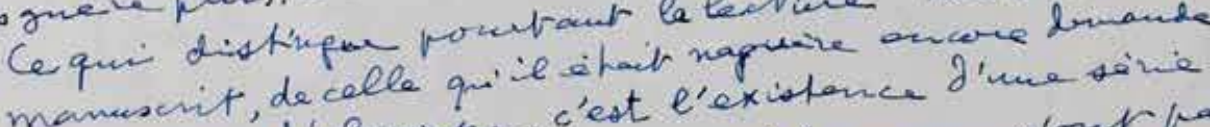
on manuscrit, de calle qu' c'est l'exiotence d'une sive

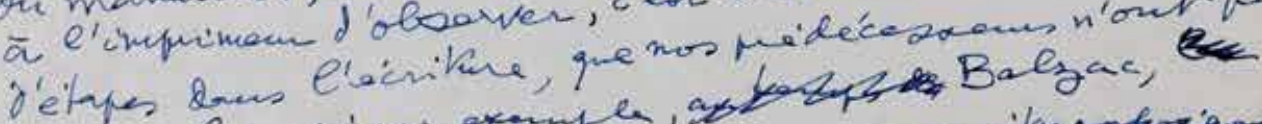

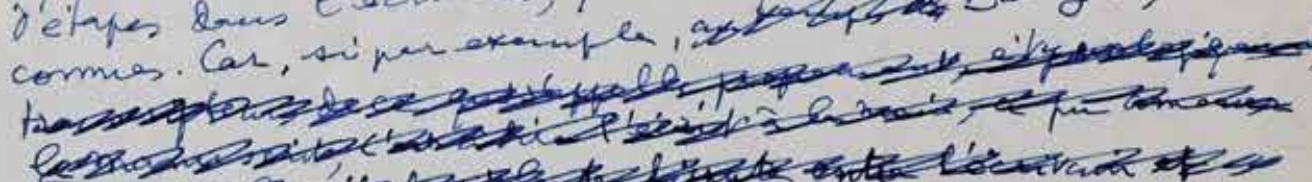

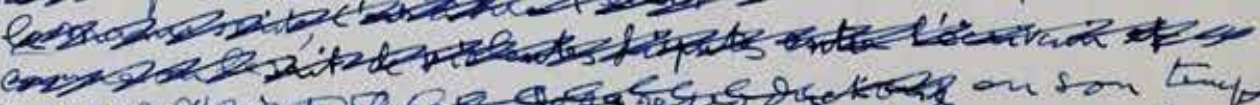

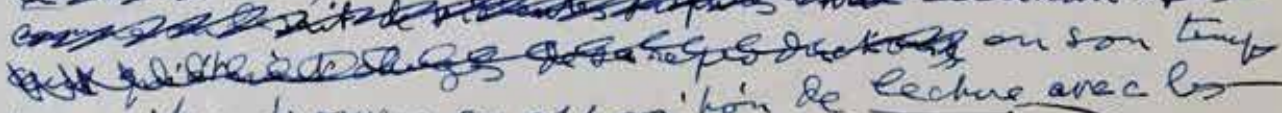
ponvait se trouner en offocitión de lechue arec $b s$

Fig. 2 : Allocution au CNRS, 4 mai 1977, version manuscrite partielle avec reprises tardives, $\mathrm{f}^{\circ}$ 5. BnF, Mss, fonds Aragon. 


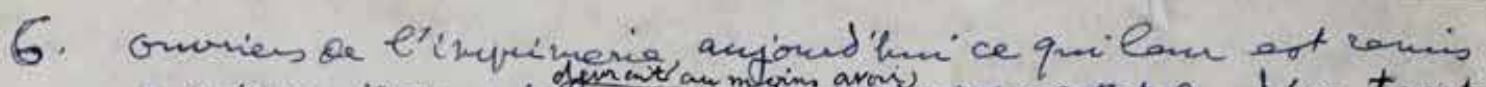

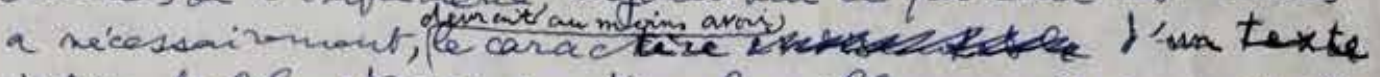
intiscubable, ot non pas d'm bronillon quí fourrait ithe mal ln, on tout an moins contesté.

Etcela, pas senlemont on fiet, que l'aubars, whme

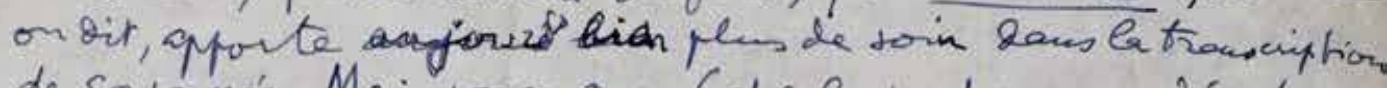
de sa pensée. Mais parce que (etcela pentramercer 'actis torts de disputs que ls hup badzaciens), cequiouremet

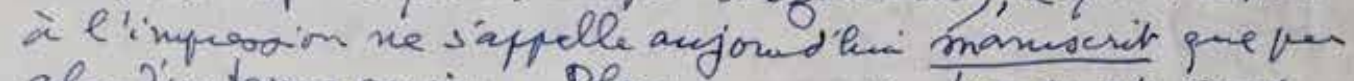
abus intem ancion... Pluspersom- he trantwer anx owies bu livre quelque chose quidoit e'crit, et ymolog juengy, à la main. De la take de l'aukeur à lacompoition ru taxte.

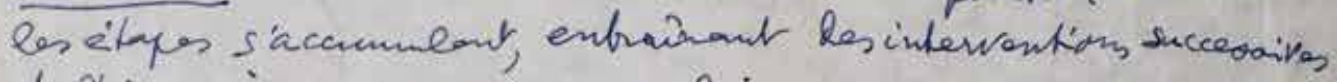

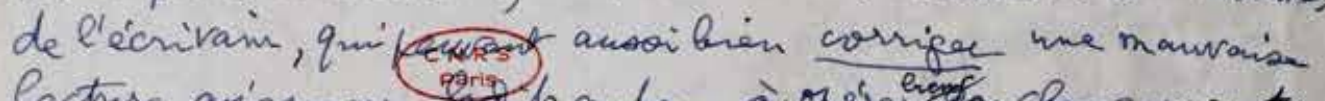
lecture quiamerou Perigh auteres à opereirgdes clangements dous la trasscriftion de sepensei.

Vour nevoyy tas bience que je voux dire...?

Parlonsens claivenent.

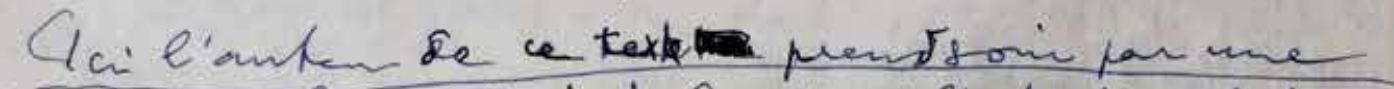

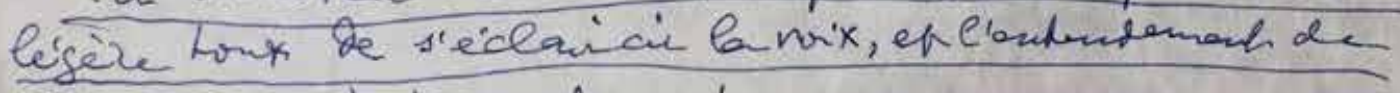
sompuleric) H(um, frum...!

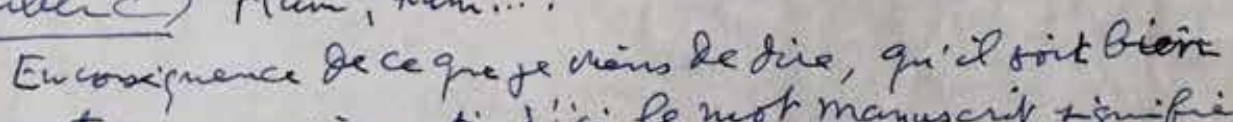
pose antrenons qu'à patii d'ici le not manuacris z gin-fie l'e'tat intemidiaie on dermei de l'objet usuellomont anisi de'signe' par ce mot, mais aussi l'arsemble, 'Sloctrowre encore

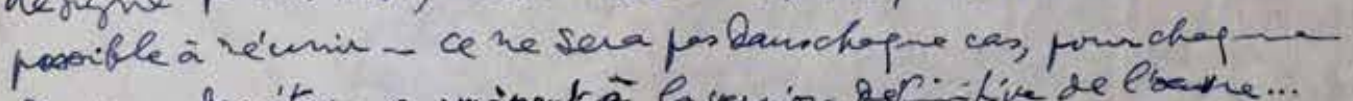
senore - desétapes qui minent à laveroion dof i Live de lbarre...

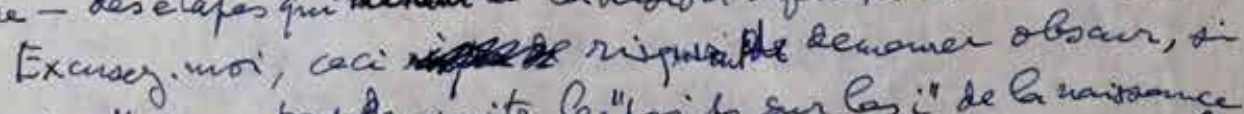
je re mettais pas toul be suite Ces" points sur bs i" de b navisance

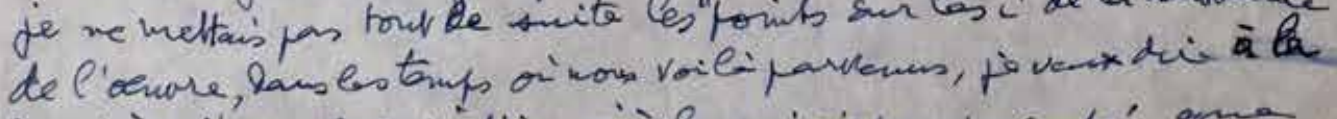

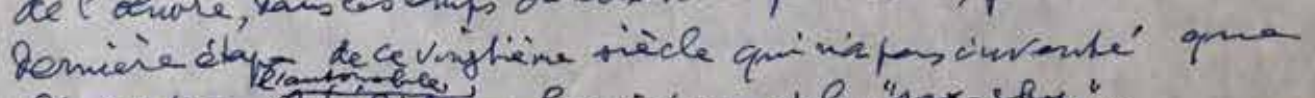
l'aviabion, feleleptiona, lecriéma, at len 'sex-shop....

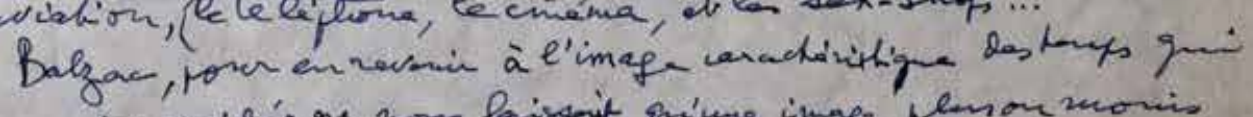

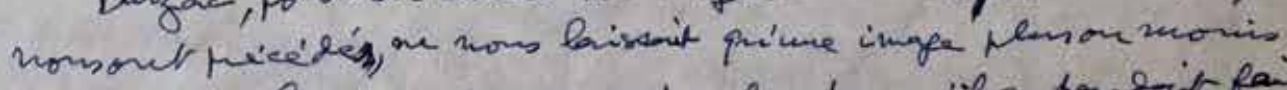

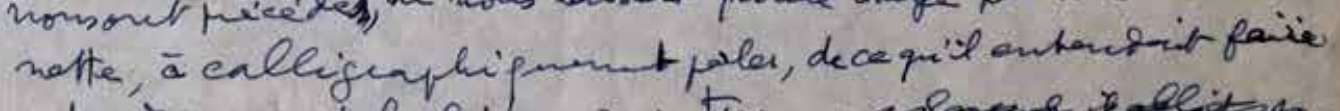

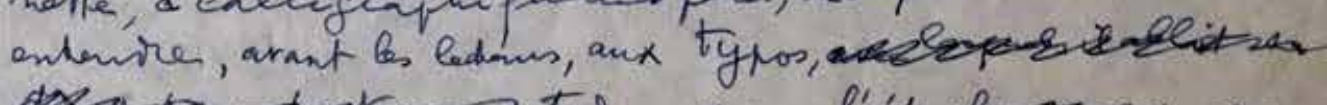

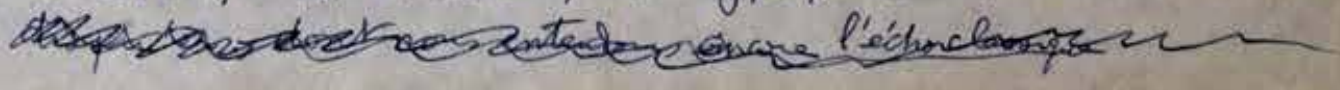

Fig. 3 : Allocution au CNRS, 4 mai 1977, version manuscrite partielle avec reprises tardives, $f^{\circ}$ 6. BnF, Mss, fonds Aragon.

(๑) Paris, BnF. Avec l'aimable autorisation de Jean Ristat 
En relevant les différentes significations du mot, Aragon pose, à raison, la question de l'authenticité 37 matérielle du manuscrit, couvrant une réalité différente de son sens étymologique, et de l'authenticité idéelle par rapport à la pensée de l'auteur. Désignant tout le travail mental qui n'apparaît pas sur le papier et l'éventuel état incomplet de l'archive - «si l'ensemble se trouve encore possible à réunir» - , il pointe cette faille que la recherche espère réduire, sinon combler, par la collaboration avec l'auteur de son vivant. Sous sa plume de préfacier on avait lu des aveux tardifs sur l'existence de faux manuscrits. La génération surréaliste était obligée de tirer presque professionnellement profit de la demande du marché d'autographes. Ce qui explique l'appréhension devant une méprise ou un abus de ses droits inaliénables par un auteur sensibilisé dès son jeune âge à la question du vrai et du faux ${ }^{38}$.

En dépit de la méfiance, la curiosité l'emporte. L'octogénaire, à l'affût de l'innovation, veut comprendre et rend compte de ce nouvel intérêt 39 pour le «texte en devenir, saisi pendant le temps de l'écriture, avec ses ratures comme ses repentirs, miroir des hésitations de l'écrivain ${ }^{40}$ ».

Concernant le contenu des archives, les évocations de l'allocution font apparaître l'impréparation. Écrivain encore actif, Aragon n'a ni préparé ou ordonné ses manuscrits ni sciemment conservé ses brouillons. Mais il connaît les grandes lignes de ses archives, qui reflètent les périodes littéraires et historiques traversées, et il assure l'auditoire d'une importante documentation à venir.

Je puis annoncer qu'en dehors des manuscrits de romans ou de livres poèmes qui sont en ma possession, je puis dire que ma donation comportera un très grand nombre d'écrits qui n'ont pas été recueillis en livres, et qui constituent des documents dont il serait peu raisonnable, pour cela, de minimiser l'importance, et pas seulement anecdotique ${ }^{41}$.

Il y aura donc de quoi faire comprendre le siècle à la postérité.

\section{Comprendre le siècle}

L'allocution est construite sur deux temps, le passé et l'avenir. Au présent, cet acte qui veut «ranger sous le titre sonore des MANUSCRITS autre chose qu'un roman par exemple, [mais] ouvrir la place à des écrits qui resteraient, si nous n'y prenions garde, des pages

\footnotetext{
37. De même qu'en parlant de la «phototypie».

38. Le nom d'Aragon dément ses ascendances véridiques. Son nom lui est effectivement propre (tout en étant taché dans le sens moral) mais faux par rapport aux deux lignées biologiques, la paternelle (Andrieux) et la maternelle (Toucas et Massillon). Voir ma contribution à Genesis, n 8, 1995 : «Quatre-vingt-dix ans de roman familial : Aragon, Quelle âme divine! ou la genèse d'une scène d'écriture», p. 51-71, en ligne sur persee.fr.

39. Pratique nouvelle par rapport à l'approche philologique et l'inventaire des variantes qui n'ont rien de nouveau pour Aragon : «Cette variante peut être [...] un sujet de réflexion pour celui à qui le manuscrit est confié, [...] mais en lui gardant strictement le caractère d'une version écartée par l'écrivain, et il est permis de se demander pourquoi. Mais guère plus. Cela n'a rien de nouveau. Ce qui distingue pourtant la lecture moderne du manuscrit, de celle qui était naguère encore demandé à l'imprimeur d'observer, c'est l'existence d'une série d'étapes dans l'écriture, que nos prédécesseurs n’ont pas connues » $\left(\mathrm{ms}^{\circ} 5\right)$.

40. Ms f ${ }^{\circ}$ 4. Et aussi : «De la tête de l'auteur à la composition du texte les étapes s'accumulent entraînant des interventions successives $\gg\left(\mathrm{ms} \mathrm{f}^{\circ} 6\right)$.

41. Ms f ${ }^{\circ} 15$.
} 
perdues dans les vents de l'histoire ${ }^{42}$ ». Deux directions s'ouvrent : comprendre, ou avoir compris, et en conséquence, faire comprendre le siècle : c'est pourquoi Aragon déclare mettre «à la disposition des chercheurs d'aujourd'hui, non pas seulement un certain nombre de manuscrits, au sens des éditeurs, mais les papiers de [sa] vie mentale, les dossiers complexes sans lesquels à [son] sens, ce qu'on appelle le manuscrit n'est que bien peu de choses, en un mot, les dossiers non pas seulement de [ses] livres, proses ou poèmes, mais tout ce qui peut éclairer les raisons de dire et d'écrire de quelqu'un comme [lui], dans ce siècle vingtième où [Aragon n'a] pas été que le soldat de deux guerres mondiales [...] et de cet autre combat [...] du nom [...] de surréalisme ${ }^{43}$ ». En effet, l'œuvre écrit les avant-gardes de son époque. Aragon avait trois ans en 1900 - par sa vie, sa création, ses aspirations et combats, il représente, à lui seul, le $\mathrm{xx}^{\mathrm{e}}$ siècle, y fait figure de paradigme. Il écrira : «Moi si j'y tenais mal mon rôle / C'était de n'y comprendre rien ${ }^{44} \gg$. Ces vers chantent sa jeunesse et disent la continuité : «comprendre», c'est ne cesser d'interroger ce qui fait énigme.

L'intention de contribuer par le legs à la compréhension de son siècle prolonge les commentaires contextualisant des récentes rééditions ${ }^{45}$. «Je ne pense pas que l'on puisse comprendre quoi que ce soit de moi, si l'on omet de dater mes pensées et mes écrits ${ }^{46}$.» Cette approche préconisée, Aragon vient de la mettre en œuvre en ce qu'il finit par nommer «[s]es œuvres posthumes 47 » par anticipation, ou dépassement, du testament. À son auditoire du CNRS, il rend compte de ce travail sur son Euvre poétique :

Puisque, de 1974 à 1977, je n'ai pu refaire le chemin de ma vie que de 1917 à 1937; c'est-à-dire le travail de vingt années seulement, donc le tiers de l'espace à franchir, et le livre actuellement à l'impression avec les années 36 et 37 laisse devant moi au moins deux fois ce que j'ai pu faire en trois ans. Verrai-je la fin de cette affaire? Même dans mes six ans que je ne suis pas sûr d'avoir devant moi [...] Et sans doute que je n'ai jamais tant écrit que cette année-ci, ce début de 1977, puisque le dernier livre avec lequel j'ai eu tant de difficultés, et dont j'ai réécrit la première année (1936) près de trois fois, va sortir ce printemps sur 464 pages 48

Aragon, en adhérant à la méthode d'analyse «scientifique» de l'histoire, avait voulu expliquer la littérature par les circonstances. Il se heurte ici au contexte historique même et doit reconnaître ses difficultés. Arrivé, entre passé et avenir, à ce moment précis de revisitation, sa vie et son œuvre lui tendent, en 1977, le miroir problématique de 1937 (et de la terreur en URSS), qui rompt ce fil de testament littéraire.

En fin de compte, ce sera à la recherche de garantir le renouvellement et la mise-au-présent, en un mot, de pérenniser l'œuvre en continuité avec ce qu'Aragon avait entrepris dans les rééditions depuis 1964 pour les romans et depuis 1974 pour la poésie. Si l'art et la littérature exaucent le désir inconscient de se survivre, Aragon, dépourvu d'ascendance, pallie par la paternité symbolique du legs l'absence de descendance.

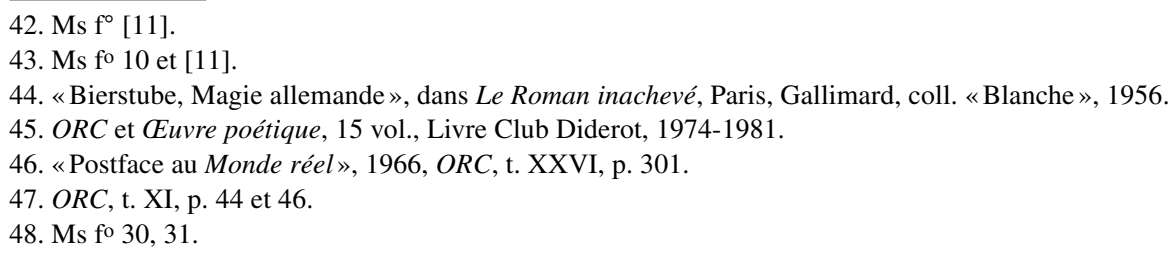




\section{Ouverture sur la relève des cadets}

«J'ai été de ceux qui, au lendemain de la Première Guerre mondiale, ont mesuré l'insuffisance de leurs prédécesseurs, à la lumière des événements de 1914 à $1918^{49}$. $\gg$ Cette observation de l'allocution caractérise, en particulier à partir de la Libération, l'attention que porte Aragon à la jeune génération 50 .

Les diverses phases de sa création sont marquées par la capacité de renouvellement. Sa poésie ne parle-t-elle pas de la curiosité pour ce qui touche au contemporain, à l'actualité, la sensibilité à l'air du temps ? Concernant les manuscrits, l'argument d'intégrer un « laboratoire » innovant devait inciter d'autres auteurs à suivre son exemple de donateur : «Je veux attirer votre attention sur le fait que si j'ai cette chance d'être le premier que vous ayez appelé [...], cette chance serait vaine si d'autres, sur les pas de l'avenir, ne venaient se joindre à moi, répondre à leur tour à votre appel ${ }^{51}$.»

Non seulement il revendique une « démarche sans précédent» 52 mais il envisage de jeter les bases de futures archives littéraires du $\mathrm{Xx}^{\mathrm{e}}$ siècle ${ }^{53}$, esquisse la voie d'enrichissements possibles, pour que le CNRS accueille ses cadets. Pratiquant à leur égard ce qu'il nomme en 1959 la «pédagogie de l'enthousiasme ${ }^{54}$ », tout comme il a «tendu la main 55 » aux jeunes créateurs, les publiant.

En souvenir et invite, il fait la récitation au CNRS, après avoir recopié douze feuillets à la main, d'un texte ancien des Lettres françaises («Le quatrième chant»), consacré aux poètes Lionel Ray, Marc Delouze et Michel Cahour (cf. note 11). Cet article est en grande partie supprimé (dix feuillets) dans les publications imprimées. Sont maintenues trois petites colonnes (deux feuillets) avec les noms de Pierre Lartigue, Maurice Regnault (sic), Jacques Roubaud et Bernard Vargaftig. Les désignant comme «[s]es enfants du Récamier», Aragon renvoie à la lecture qui les avait réunis, alors jeunes poètes âgés de la trentaine, au théâtre Récamier dont il avait loué la salle pour la soirée du 14 décembre 196556. Pierre Lartigue écrira à ce propos : «Par l'attention qu'il nous porte (ainsi qu'à beaucoup d'autres) Aragon affirme que la poésie existe et existera tant que vivra la langue ${ }^{57}$.»

En conclusion de l'allocution, la présentation du poète Jean Ristat, d'une génération plus récente que les «enfants du Récamier», témoigne du même enthousiasme et culmine

49. Ms f 12

50. Depuis 1931, années de la mort de son père, et plus directement à partir de 1942 et le décès de sa mère, Aragon accède à la génération symboliquement parentale.

51. $\mathrm{Ms} \mathrm{f}^{\circ} 13$.

52. $\mathrm{Ms} \mathrm{f}^{\circ} 4$.

53. Institution faisant défaut en France à l'époque. La lacune a été réparée entre-temps par la création de l'Institut mémoires de l'édition contemporaine (IMEC) en 1988.

54. Cette pédagogie implique l'accueil enthousiaste des jeunes auteurs. J'abats..., op. cit., p. 110.

55. $\mathrm{Ms} \mathrm{f}^{\circ} 12$. Aragon détourne la formule de «la main tendue» (à la population catholique) de Maurice Thorez, 1936.

56. Aragon se trompe d'année («1971»), pas de nombre : «Ils étaient six, si je sais encore compter» $\left(\mathrm{ms} \mathrm{f}^{\circ} 10\right)$. Mais, plus loin, il oublie les noms de Jacques Garelli et André Libérati dans le récit de la soirée du Récamier, ajoutant par erreur «ceux qui doivent leur premier jour à Elsa. (Roubaud donc qui avait alors quinze ans, Dominique Tron qui en avait quatorze, et [Claude] Adelen... vous vous rappelez, Adelen l'autre année ?)» (ms fo 27). Récemment, Claude Adelen a témoigné de sa présence dans l'auditoire («Préface » à Pierre Lartigue, Des poèmes comme des îles, Caluire-et-Cuire, Sous le Sceau du Tabellion, 2019, p. 5).

57. Pierre Lartigue, Un soir, Aragon..., Paris, Les Belles Lettres, 1995, p. 13. 
en intronisation du «prolongateur» d'Aragon : «Jean Ristat, que je connais, je crois, depuis treize ans, est déjà pour moi l'image même de l'avenir [...] je le reconnais ici publiquement pour bien mieux qu'un collaborateur... 58 ».

Jalon historique, la cérémonie au CNRS donne forme purement symbolique au fonds Aragon et l'Allocution peint les archives en désordre vivant. Aragon annonce : «La diaspora de mes anciennes pensées, la dispersion de mes écrits, ce qu'il m'en reste au moins, et c'est comme un monde à tirer de l'enfer... 59 ». Seul le regard de l'autre peut mesurer ce «monde» et estimer son ampleur : «mes amis me disent» ou «ceux qui me connaissent bien disent qu'il faudrait des camions pour les mettre à vos pieds $60 »$, ces manuscrits. Il renvoie donc au reflet spéculaire bien connu61, reflet qui prend ici la forme d'une passation de pouvoir et remet à autrui la suite. Serait-ce à présent un doute sur la démarche endogène, la grande entreprise de réédition de ses œuvres commentées par lui-même, dites « œuvres posthumes »? L'inquiétude de ne pouvoir mener à terme la gestion et le classement des manuscrits s'exprime ici, dans une phrase sans fin du «Post-scriptum» à la fois clôturant et ouvrant sur l'avenir du fonds. Phrase en quête de son sujet, grammatical et sujet écrivant, quête dont la raison, ou le fond(ement), est la vie même liée intimement à ces papiers.

Quand on aura pu réunir, avec moi de mon vivant, et s'il ne m'est pas donné dans ce qu'il me reste à vivre d'accomplir dans son entier le travail auquel vous m'avez appelé, peut-être bien sans vous rendre pleinement compte de l'énormité de la tâche, que demande le rassemblement, le classement, des manuscrits, œuvres et documents, que j'ai accumulés pendant les trois premiers quarts du vingtième siècle, s'il ne m'est pas donné dis-je, d'accomplir moi-même ce travail, même aidé par quelqu'un dont je vous parlerai plus tard, et dont la présence à mes côtés est la chance de mes derniers jours et d'une survie que je lui devrai... je tiens à dire aujourd'hui que ceci s'inscrit naturellement dans l'ordre des préoccupations majeures de mon existence. Et si je n'arrive pas aujourd'hui, les bras chargés de paperasses, par la longueur même de ce discours et sa démesure, je tiens à ce que vous compreniez bien que je me refuse ainsi à une hâtive parade, qui ne serait au bout du compte que de la comédie... parce que le rassemblement des feuilles qui constituent non seulement mes œuvres, mais aussi tout ce que j'ai pu et pourrai retrouver de leurs ébauches, le témoignage de mes variations au cours même de leur écriture, c'est-à-dire tout ce qui donnera aux chercheurs en question le matériel de ce que les livres achevés ne suffisent pas à leur faire connaître, comprendre, et utiliser pour une étude qui ne peut se borner, quel qu'il soit, à un écrivain, mais l'expérience et la connaissance de notre temps, par la voie de l'écrit, par la diversité des écrivains et de leurs expériences $62 \ldots$

Ce passage s'ouvre comme une porte battante sur l'avenir - pour citer son auteur - et inscrit en son centre le grand et définitif vide de l'(in)achèvement. Car la cérémonie du

58. Ms fo 29-32. «[...] si je puis me permettre de lui donner ce nom, le prolongateur de ce qui aura été ma vie, ma raison d'être, mon métier» $\left(\mathrm{ms} \mathrm{f}^{\circ} 31\right)$.

59. $\mathrm{Ms} \mathrm{f}^{\circ} 10$.

60. Ms fo 13,14 .

61. Connu de La Mise à mort (1965) et du «croisement» de ses romans avec ceux d'Elsa Triolet (ORC). Dans les commentaires, les deux écrivains établissent des relations entre leurs romans respectifs.

62. Ms fo 12,13

\section{La phrase sans fin}


CNRS et le legs concrétisent aussi cette mesure conservatoire, initiée par d'autres, d'assurer cet «au-delà de l'œuvre» qui n'est plus ici une image ludique d'art romanesque ${ }^{63}$, afin de l'ouvrir à l'étude et au commentaire exogènes.

L'élaboration du futur fonds sera réalisée par le «prolongateur», à qui l'auteur ne doit rien moins que sa vie :

C'est il y a trois ans, et un peu plus de cela, aux derniers jours de janvier 1974, que, sur la place de la Concorde, j' avais perdu pour quelques heures le sentiment de vivre [...] les médecins parmi lesquels je me réveillai, m'avaient cru mort. Je n'en avais aucune idée, et je ne compris que dans le regard d'un jeune homme dont j'ai ici à vous parler.

Car il s'agit de Jean Ristat, dont, quatre mois plus tard, le nom allait apparaître dans ce premier volume de ma folie, je dis bien : de ma folie, puisqu'encore dans les plâtres, immobilisé dans mon lit, j'avais, oui : follement accepté d'écrire sous ce titre L'CEuvre poétique, une collection de volumes que je n'aurais jamais laissé entreprendre sans l'aide de Ristat ${ }^{64}$.

Cet autre, implant au cœur du «Post-scriptum», de Ce-qui-sera-écrit-après..., renouvelle la vie sauvée d'un accident, telle qu'elle avait été sauvée jadis dans des conditions comparables : Elsa Triolet, «sans qui je me serais tu», écrivait Aragon, et insiste ensuite sur l'homophonie, ou presque, de cette dédicace avec sans qui je me serais tué. L'analogie est double; entre l'accident et le suicide, entre le corps et le corpus : à qui on doit la vie, on désire croiser avec lui son œuvre et/ou lui confier l'édition de ses œuvres, de sa succession, ce qui sera le cas de Jean Ristat. Entre deux personnes grammaticales, «je» et «on», l'Autre est institué en lieu et place du Sujet. C'est à l'autre, le passeur, que revient d'achever ou «d'accomplir dans son entier le travail auquel vous m'avez appelé», vous, auditoire ou acteurs qui sollicitent, vous, public de lecteurs, collectivité nationale, qui confèrent son statut social à la création et au créateur.

À la mort d'Aragon, en décembre 1982, la première partie des archives peuple le coffrefort 65 . Mais le légateur n'a pas pu rassembler son fonds ni, encore moins, répondre aux questions que les chercheurs espéraient lui poser. Quelque temps après cet acte au CNRS, un de ses derniers gestes publics, Aragon est affecté d'une crise délirante. Il ne reviendra pas à son Euvre poétique ${ }^{66}$. La postérité rencontrera, dans les papiers par-ci par-là, le gros stylo-feutre noir, témoin d'interventions autographes tardives. L'instrument adapté à la vue faiblissante retrace quelques manuscrits ternis, écrit de dernières notes, et nous adresse des remarques telles que celle sur le bloc-notes d'avant-texte des années 1958-1959 (fig. 4).

63. «C'est l'au-delà qui m'intéresse », écrit Aragon à propos du roman.

64. Ms fo 29,30 .

65. Jean Ristat transfère cette partie au 61, rue de Richelieu, Paris II ${ }^{\mathrm{e}}$. Sous la direction de M. Apel-Muller, chargé des manuscrits d'Elsa Triolet sur le contrat du legs, seront entreprises deux campagnes de rassemblement en 1985, l'une au domicile parisien d'Aragon, l'autre dans sa maison de campagne.

66. Jean Ristat finit l'édition, édite les dernières publications et témoigne : Aragon déchire ce qui lui semblait devoir être soustrait aux regards. 


\section{Bci, eainsy quejezospina...}

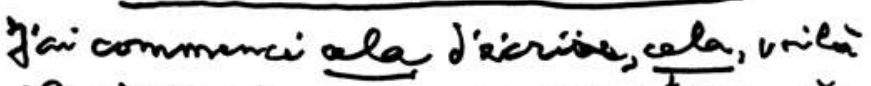

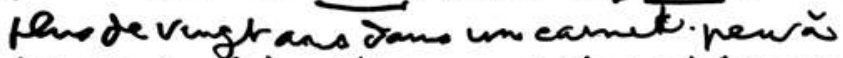
few quise deimolit commanimpontrimper

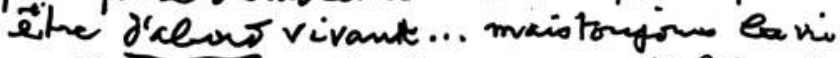

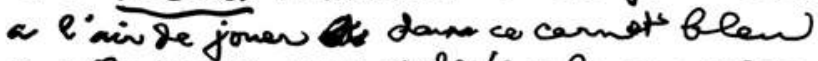

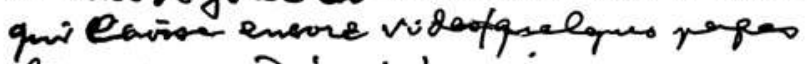
blawchs, on di snis trop vienx pour ce cache-epole ats afformbielonis...

\section{Ding dow .... Ding don... A thases youtow de roin rawge ow bland ....}

Ici, laissez que je respire...

J'ai commencé cela d'écrire, cela, voilà plus de vingt ans dans un carnet peu à peu qui se démolit comme n'importe quel être d'abord vivant... mais toujours la vie a l'air de jouer dans ce carnet bleu qui laisse encore vides quelques pages blanches, où je suis trop vieux pour ce cache-cache et ce tourbillon...

\section{[dessin de cloche]}

Ding don... Ding don... À chacun son tour de voir rouge ou blanc...

Fig. 4 : Ajout manuscrit tardif sur bloc-notes en toile plastifiée bleue, $\mathrm{f}^{\circ} 48$. $\mathrm{BnF}, \mathrm{Mss}$, fonds Aragon.

() Paris, BnF. Avec l'aimable autorisation Jean Ristat 
RENATE LANCE-OTterbein, retraitée du CNRS, a collaboré au sein du CAM, puis de l'ITEM à l'équipe Heine et à la Codicologie (1976-1982). Elle a travaillé au fonds Elsa Triolet-Aragon (de 1982 à 2002) : inventaire et relevés, classement, catalogue (Le Fonds Elsa Triolet-Aragon du CNRS, un legs à la nation française, catalogue remis à la direction du CNRS en 1996 et consultable au département des Manuscrits, BnF), publications en revues, thèse. Elle traduit aujourd'hui (en collaboration avec Alain Lance) les œuvres de Christa Wolf, Volker Braun et Ingo Schulze.

\section{Résumés \\ Un fonds, son legs}

En 1977, Aragon confie à la recherche (ITEM / CNRS) le «legs littéraire d'Elsa Triolet et l'ensemble des manuscrits et documents en sa possession». Dans son allocution inaugurale, il interroge les notions de «manuscrit» et de «recherche», constate l'évolution du sens et des méthodes et salue l'innovation de la critique génétique qui pousse la curiosité jusqu'à l'étude des brouillons. Ailleurs, il avait réclamé le droit de l'écrivain et créateur à la recherche. À présent, souhaitant faire comprendre ce siècle dans lequel il fut inventeur d'avant-gardes et de mouvements progressistes, il accepte que la recherche s'empare de ses archives et incite à les enrichir avec des auteurs cadets qu'il avait encouragés et publiés. L'un d'eux, Jean Ristat, est présenté comme successeur. Le manuscrit de l'allocution permet de mieux mesurer la préoccupation de l'octogénaire au sujet de cette ouverture sur l'avenir : Aragon, demandant sérieux et discrétion, délègue à la postérité le rayonnement de son œuvre.

In 1977 Aragon entrusted to the CNRS (ITEM) the "Elsa Triolet's literary legacy and all the manuscripts and documents in his possession". In his inaugural speech he questioned the notions of "manuscript" and "research", realising the evolution of the meaning and methods, and acknowledged the innovation represented by critical genetics that drives curiosity to study drafts. Elsewhere he had claimed the writer's right and creator to do research. At this point, wishing to explain this century in which he was an inventor of avant-gardes and of progressive movements, he accepted that the research community take control of his archives and also incited researchers to enrich his archives with the manuscripts of younger writers, whom he had encouraged and published. One of them, Jean Ristat, was presented as his successor. The speech's manuscript allows us to better measure the octogernarian's preoccupation concerning this future path: Aragon, wishing for seriousness and discretion, appointed posterity with the perpetuation of his work.

1977 betraute Aragon die wissenschaftliche Forschung (ITEM / CNRS) mit dem ,literarischen Erbe von Elsa Triolet und allen in seinem Besitz befindlichen Manuskripten und Dokumenten“. In seiner Antrittsrede hinterfragt er die Begriffe „Manuskript“ und „Forschung“ und stellt fest, dass sich die Begriffe und ihre Methoden wandeln. Er begrüßt als Neuheit der textgenetischen Forschung, dass ihre Neugier bis in Handschriften, ja sogar Entwürfe vordringt. An anderer Stelle war er dafür eingetreten, die Arbeit der Schriftsteller und Künstler als Forschen zu bezeichnen. Nun akzeptiert er zum Verständnis seines Jahrhunderts, in dem er Erfinder von Avantgarden und fortschrittlichen Bewegungen war, dass sich die Forschung seines Nachlasses annimmt und regt an, ihn mit jüngeren Autoren zu bereichern, die er ermutigt und veröffentlicht hatte. Einer von ihnen, Jean Ristat, wird als Nachfolger vorgestellt. Die Handschrift der Rede zeigt einen in die zukünftige Rezeption seines Werkes gerichteten Achtzigjährigen; nicht ohne an Ernsthaftigkeit und Diskretion zu appellieren, übergibt Aragon der Nachwelt die Resonanz seines Werkes.
En 1977, Aragon confía a la investigación (ITEM/CNRS) el "legado literario de Elsa Triolet y el conjunto de los manuscritos y documentos en su posesión". En su discurso inaugural, el escritor se interroga acerca de las nociones de "manuscrito" y de "investigación", constata la evolución del sentido y de los métodos y celebra la innovación de la crítica genética que extiende su curiosidad hasta el estudio de los borradores. En otra ocasión, había reivindicado el derecho del escritor y del creador a la investigación. Ahora, tratando de tornar comprensible ese siglo en el cual fue inventor de vanguardias y movimientos progresistas, acepta que la investigación se apodere de sus archivos, incitando a enriquecerlos con los autores más jóvenes, que él mismo había alentado y publicado. Uno de ellos, Jean Ristat, es presentado como sucesor. El manuscrito de este discurso, permite apreciar más cabalmente la preocupación del octogenario por esta apertura hacia el futuro: Aragon, exigiendo seriedad y discreción, delega a la posteridad la resonancia de su obra.

Em 1977, Aragon confia à pesquisa (ITEM/CNRS) o «legado literário de Elsa Triolet e o conjunto de manuscritos e documentos que ela possuia». Em seu discurso inaugural, ele questiona as noções de «manuscrito » e de "pesquisa», consta a evolução do sentido e dos métodos e congratula-se com a novidade da crítica genética, que leva a curiosidade até ao estudo dos rascunhos. Já, em outros lugares, ele havia reclamado o direito dos escritores e dos criadores a serem objeto de pesquisa. Agora, desejando tornar compreensível um século em que ele foi inventor de vanguardas e de movimentos progressistas, ele aceita que a pesquisa se apodere de seus arquivos e incita a enriquecê-los com escritores mais novos que ele havia encorajado e publicado. Um deles, Jean Ristat, é apresentado como sucessor. $\mathrm{O}$ manuscrito do discurso permite determinar melhor a preocupação do octogenário com a questão desta abertura ao porvir: Aragon, pedindo seriedade e discrição, delega à posteridade a projeção de sua obra.

Nel 1977 Aragon affida alla ricerca (ITEM / CNRS) il "lascito letterario di Elsa Triolet e l'insieme dei manoscritti e documenti in suo possesso". Nel discorso inaugurale egli interroga le nozioni di "manoscritto" e "ricerca", constata l'evoluzione del senso e delle metodologie e rende omaggio all'innovazione della critica genetica che spinge la curiosità fino allo studio degli scartafacci. Aragon aveva già rivendicato il diritto dello scrittore e del creatore alla ricerca. Desiderando far comprendere questo secolo in cui fu inventore di avanguardie e movimenti progressisti, egli accetta ora che la ricerca si impossessi dei suoi archivi, incitando ad arricchirli con altri di autori più giovani che egli aveva incoraggiato $\mathrm{e}$ pubblicato. Uno di questi, Jean Ristat, è presentato come il suo successore. Il manoscritto del discorso inaugurale permette di valutare meglio la preoccupazione dell'ottuagenario riguardo quest' apertura sull'avvenire: chiedendo serietà e discrezione, Aragon delega alla posterità la trasmissione della sua opera. 